\title{
X-RAY EMISSION FROM WOLF-RAYET STARS
}

Pointed ROSAT PSPC observations of nine single WN stars

\section{U. WESSOLOWSKI, W.-R. HAMANN and L. KOESTERKE Institut für Theoretische Physik und Sternwarte der Universität Olshausenstraße 40, D-24098 Kiel, B.R. Deutschland}

D.J. HILLIER
Department of Physics \& Astronomy, University of Pittsburgh, PA, U.S.A.

\author{
and
}

J. PULS

Institut für Astronomie \& Astrophysik, Universitätssternwarte München, B.R.D.

\begin{abstract}
Results from pointed ROSAT PSPC observations of nine single WN-type Wolf-Rayet stars are presented. Spectra of sufficient quality were obtained for two of them (WR1, WR110). The long exposure $(35.5 \mathrm{ksec}) \mathrm{X}$-ray spectrum of WR1 is more closely investigated with a semi-empirical model developed by Baum et al. (1992).
\end{abstract}

Among the 48 Wolf-Rayet (WR) stars observed with the IPC of the EINSTEIN observatory about one third showed detectable X-ray flux (Pollock 1987). An improvement in sensitivity together with moderate spectral information ( $4-5$ independent energy bands) has been offered recently by the PSPC

TABLE I

Pointed ROSAT PSPC observations of 9 single WN-type WR stars.

\begin{tabular}{|c|c|c|c|c|c|c|c|}
\hline \multirow[b]{2}{*}{ WR } & \multirow[b]{2}{*}{$\begin{array}{l}\text { spectral } \\
\text { type }\end{array}$} & \multirow[b]{2}{*}{ HD } & \multicolumn{4}{|c|}{$\longrightarrow$} & \multirow{2}{*}{$\begin{array}{c}\text { EINSTEIN } \\
\text { IPC rate } \\
\left(\mathrm{ksec}^{-1}\right)\end{array}$} \\
\hline & & & $\begin{array}{l}\text { proposal } \\
\text { ID }\end{array}$ & $\begin{array}{r}t_{\text {obs }} \\
(\mathrm{sec})\end{array}$ & counts & $\begin{array}{c}\text { rate } \\
\left(\mathrm{ksec}^{-1}\right)\end{array}$ & \\
\hline \multirow[t]{2}{*}{1} & WN5-s & 4004 & 201272 & 8399 & $245 \pm 16$ & 29.2 & - \\
\hline & & & 201535 & 27091 & $873 \pm 30$ & 32.2 & \\
\hline 2 & WN2-w & 6327 & 200720 & 9729 & $60 \pm 9$ & 6.1 & - \\
\hline 7 & WN4-s & 56925 & 200718 & 7346 & $17 \pm 5$ & 2.3 & - \\
\hline 16 & WN8 & 86161 & 200715 & 7805 & $<2.3$ & $<0.3$ & $4(1-8)$ \\
\hline \multirow[t]{2}{*}{46} & WN3p-w & 104994 & 201271 & 826 & $7 \pm 3$ & 8.5 & $4(2-7)$ \\
\hline & & & 201271-1 & 7830 & $66 \pm 9$ & 8.4 & \\
\hline 78 & WN7 & 151932 & 200716 & 10421 & $\lesssim 24$ & $\lesssim 2.3$ & $5(1-8)$ \\
\hline 110 & WN6-s & 165688 & 200717 & 8430 & $15 \tilde{4} \pm 14$ & 18.3 & - \\
\hline 152 & WN3-w & 211564 & 201275 & 4960 & $13 \pm 5$ & 2.7 & $4(0-8)$ \\
\hline 157 & WN4.5-w & 219460 & 201273 & 5856 & $\lesssim 7$ & $\lesssim 1.2$ & - \\
\hline
\end{tabular}




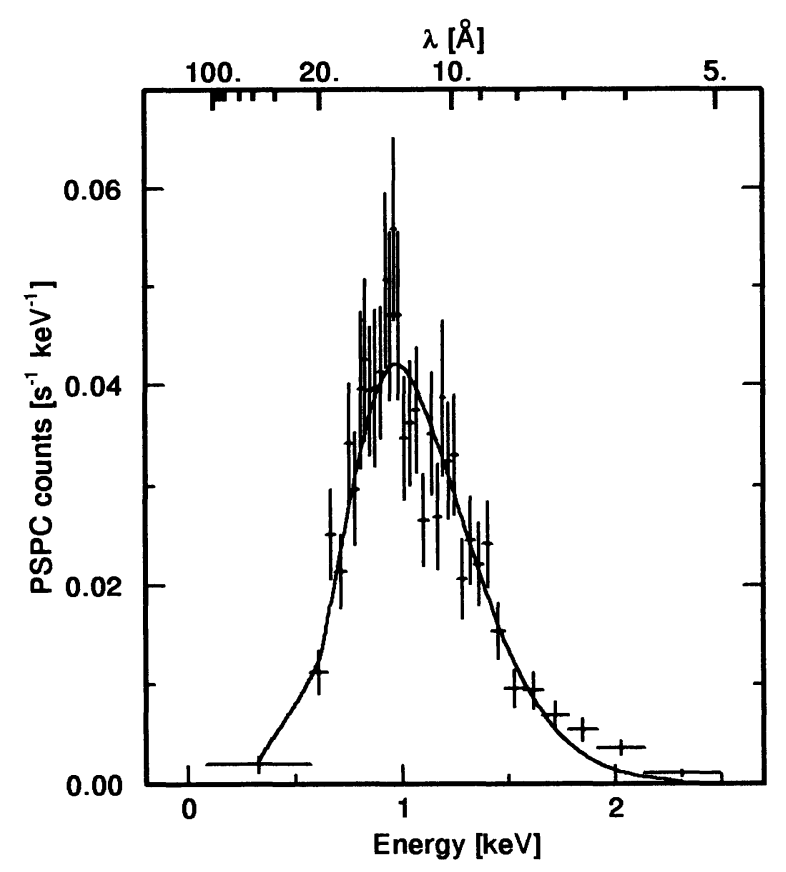

Fig. 1. Merged (35.5 ksec total exposure time, binned to $\mathrm{S} / \mathrm{N}=5$ ) ROSAT PSPC observation of WR 1 (WN 5-s), compared to a semi-empirical two-component model (cf. Baum et al. 1992). The hot component producing the $X$-rays has a temperature of $T_{X}=210^{6} \mathrm{~K}$ and an exceptionally high filling factor of $X_{\text {fill }}=20 \%$, while the normal gas component is in radiative equilibrium. Fitting the count rate spectrum with the EXSAS tool FIT/SPECTRUM yields a column density of $N_{\mathrm{H}}=2.510^{21} \mathrm{~cm}^{-2}$ for the interstellar absorption.

Results for our sample of nine (putatively) single WR stars covering nearly the whole range of WN subtypes are compiled in Table 1. Spectra of sufficient quality were obtained only for WR1 and WR110.

We merged the two pointed observations of WR 1 and we investigated the X-ray spectrum with a semi-empirical model developed by Baum etal. (1992). The observed spectral distribution of the X-rays can be reproduced with that model for reasonable choices of $T_{\mathrm{X}}$ and $X_{\text {fill }}$ (cf. Fig.1). The set of stellar parameters which simultaneously allows us to fit the observed line spectrum is $T_{*}=55 \mathrm{kK}, R_{*}=3.5 \mathrm{R}_{\odot}, \dot{M}=10^{-4.2} \mathrm{M}_{\odot} / \mathrm{yr}, v_{\infty}=2000 \mathrm{~km} / \mathrm{s}$, and a nitrogen abundance of $\beta_{\mathrm{N}}=1.5 \%$ (by mass).

The "standard model" for WR atmospheres, although neglecting the Xray emitting hot component, remains valid for describing the visual and UV spectrum.

\section{References}

Baum E., Hamann, W.-R., Koesterke, L., Wessolowski, U. 1992, $A \& A$ 266, 402 Pollock, A.M.T. 1987, ApJ 320, 283 